S. ROPPONGI

KODAI MATH. J.

18 (1995), 44-56

\title{
ON SINGULAR SOLUTIONS FOR A SEMILINEAR ELLIPTIC EQUATION
}

\author{
SUSUMU ROPPONGI
}

\section{Introduction}

Let $\Omega$ be a bounded domain in $\boldsymbol{R}^{n}(n \geqq 2)$ with smooth boundary $\partial \Omega$. And let $\Sigma$ be a $C^{\infty}$-compact submanifold of $\Omega$ of dimension $m(0 \leqq m \leqq n-1)$. We take an arbitrary $\alpha(x) \in C^{\infty}(\Sigma)$ such that $\alpha(x)>0$ on $\Sigma$ and consider the following equation.

$$
\left\{\begin{array}{l}
-\Delta u=u^{p}+\alpha \delta_{\Sigma} \quad \text { in } \mathscr{D}^{\prime}(\Omega) \quad(p>1) \\
0 \leqq u \in C^{2}(\Omega \backslash \Sigma),
\end{array}\right.
$$

where $\delta_{\Sigma}$ is the measure defined by

$$
\left\langle\delta_{\Sigma}, \eta\right\rangle=\int_{\Sigma} \eta(\sigma) d \sigma
$$

for any $\eta \in C_{0}^{\infty}(\Omega)$.

What can one say about the existence of a solution of (1.1) and the local behaviour of its solution near $\Sigma$ ? We have the following.

THEOREM 1. There exists a solution of (1.1) if and only of $1<p<(n-m) /$ $(n-m-2)(1<p<\infty$ if $n-m \leqq 2)$. And there exists a solution $u$ of (1.1) satisfying

$$
\left\{\begin{array}{l}
\left.C_{1} d(x)^{-(n-m-2)} \leqq u(x) \leqq C_{2} d(x)^{-(n-m-2)} \quad \text { near } \Sigma \text { (if } m \leqq n-3\right) \\
C_{1}|\log d(x)| \leqq u(x) \leqq C_{2}|\log d(x)| \quad \text { near } \Sigma(\text { if } m=n-2) \\
\left.u(x) \in C^{0}(\Omega) \quad \text { if } m=n-1\right),
\end{array}\right.
$$

where $d(x)$ denotes the distance between $x$ and $\Sigma$. Here $C_{1}$ and $C_{2}$ denote some positive constants.

THEOREM 2. Assume that $p<n /(n-2)(p<\infty$ if $n=2)$. Then the same bounds as in (1.3) hold for any $u$ satisfying (1.1) and, in addition,

$$
u \in L_{\mathrm{loc}}^{p}(\Omega) \quad \text { if } m=n-1 .
$$

Received May 2, 1992 ; revised February 21, 1994. 
Remarks. 1. It is curious to the author that the exponent $p$ such that (1.1) has a solution depends on the dimension of $\Sigma$. When $\Sigma=$ a point $\}$, Lions [6] has proved the above results. Therefore we may assume that $m \geqq 1$ hereafter. When $p \in(1,(n+2) /(n-2))(n \geqq 3)$, the following holds immediately from Gidas and Spruck [5, Theorem 3.1, pp. 540-541], since $-\Delta u=u^{p}$ in $\Omega \backslash \Sigma$.

$$
u(x) \leqq C_{2} d(x)^{-2 /(p-1)} \quad \text { near } \Sigma
$$

Since $p<(n-m) /(n-m-2)$, the upper bounds in (1.3) is more sharp than that in (1.5). When $p \geqq n /(n-2)$, we do not know the more sharp estimates than (1.5). Furthermore, when $p \geqq(n+2) /(n-2)$, we do not know the behaviour of the solution of (1.1) in general.

2. In the case $m \leqq n-2, u \in L_{\mathrm{loc}}^{p}(\Omega)$ holds for any $u$ satisfying (1.1) [see Lemma 2.1 in section 2]. We suspect that the assumption (1.4) is not necessary.

3. For the related papers we see Aviles [1], Brezis and Lions [3], Gidas and Spruck [5], Serrin [7] and the references in the above papers.

The other case where $u^{p}$ is replaced by $-|u|^{p-1} u$ is discussed in, for example, Brezis and Veron [4], Vazquez and Veron [8], Veron [9], [10].

\section{Asymptotics}

Let $\Omega, \Sigma, \alpha(x)$ be as before. Let $G(x, y)$ be the Green function of $-\Delta$ in $\Omega$ associated with the Dirichlet boundary condition. Then,

where $S(x, y) \in C^{\infty}(\Omega \times \Omega)$ and

$$
G(x, y)-S(x, y)=\left\{\begin{array}{l}
K_{n}|x-y|^{2-n} \quad(\text { if } n \geqq 3) \\
-(1 / 2 \pi) \log |x-y| \quad \text { (if } n=2),
\end{array}\right.
$$

$$
K_{n}=\left((n-2)\left|S^{n-1}\right|\right)^{-1} \quad(n \geqq 3) .
$$

Here $\left|S^{n-1}\right|$ denotes the surface area of the unit sphere of $\boldsymbol{R}^{n}$.

We put

$$
g(x)=\int_{\Sigma} G(x, \sigma) \alpha(\sigma) d \sigma .
$$

Then $0 \leqq g \in C^{\infty}(\bar{\Omega} \backslash \Sigma)$ and $g$ satisfies

$$
\begin{cases}-\Delta g=\alpha \delta_{\Sigma} & \text { in } \mathscr{D}^{\prime}(\Omega) \\ g=0 & \text { on } \partial \Omega\end{cases}
$$

By Propositions A.2 and A.3 in Appendix, we have

$$
\left\{\begin{aligned}
C_{1} d(x)^{-(n-m-2)}-D_{1} \leqq g(x) \leqq C_{2} d(x)^{-(n-m-2)} & \text { (if } m \leqq n-3) \\
C_{1}|\log d(x)|-D_{1} \leqq g(x) \leqq C_{2}|\log d(x)|+D_{2} & \text { (if } m=n-2) \\
g(x) & \in C^{0}(\bar{\Omega}) \quad(\text { if } m=n-1)
\end{aligned}\right.
$$




$$
\begin{gathered}
\left\{\begin{array}{l}
\left.g \in L_{\mathrm{loc}}^{q}(\Omega) \quad \text { for } q \in[1,(n-m) /(n-m-2)) \quad \text { (if } m \leqq n-3\right) \\
\left.g \notin L_{\mathrm{loc}}^{q}(\Omega) \quad \text { for } q \in[(n-m) /(n-m-2), \infty) \quad \text { (if } m \leqq n-3\right)
\end{array}\right. \\
g \in L_{\mathrm{loc}}^{q}(\Omega) \quad \text { for } q \in[1, \infty) \quad(\text { if } m=n-2) .
\end{gathered}
$$

Here $C_{1}, C_{2}, D_{1}$ and $D_{2}$ denote some positive constants.

LEMma 2.1. Assume that $m \leqq n-2$ and $u$ satisfies

$$
\left\{\begin{array}{l}
-\Delta u=u^{p} \quad \text { in } \Omega \backslash \Sigma \quad(p>1) \\
0 \leqq u \in C^{2}(\Omega \backslash \Sigma) .
\end{array}\right.
$$

Then $u \in L_{\mathrm{loc}}^{p}(\Omega)$.

Proof. We take a $C^{\infty}$-convex function $\Phi$ on $[0, \infty)$ such that $\Phi(0)=1, \Phi(t)$ $=0$ for $t \geqq 1$ and put

$$
\xi_{\varepsilon}(x)=\left\{\begin{array}{lr}
\Phi\left(C_{1}^{-1} \varepsilon^{n-m-2}\left(g(x)+D_{1}\right)\right) & (\text { if } m \leqq n-3) \\
\Phi\left(C_{1}^{-1}|\log \varepsilon|^{-1}\left(g(x)+D_{1}\right)\right) & (\text { if } m=n-2) .
\end{array}\right.
$$

Here $C_{1}$ and $D_{1}$ denote the same constants as in (2.3). Then, by (2.2) and (2.3), we can easily get

$$
\begin{array}{ll} 
& \xi_{\varepsilon}(x) \in C^{\infty}(\Omega), \quad 0 \leqq \xi_{\varepsilon}(x) \leqq 1 \quad \text { on } \Omega \\
\xi_{\varepsilon}(x) \rightarrow 1 & \text { as } \varepsilon \rightarrow 0 \quad \text { a. e. in } \Omega \\
\xi_{\varepsilon}(x)=0 & \text { if } d(x)<\varepsilon \\
\nabla \xi_{\varepsilon}(x) \rightarrow 0 & \text { as } \varepsilon \rightarrow 0, \text { uniformly on any compact subsets of } \Omega \backslash \Sigma \\
\Delta \xi_{\varepsilon}(x) \geqq 0 & \text { a.e. in } \Omega .
\end{array}
$$

Let $\eta \in C_{0}^{\infty}(\Omega)$ such that $0 \leqq \eta \leqq 1, \eta=1$ near $\Sigma$. Since $\eta \xi_{\varepsilon} \in C_{0}^{\infty}(\Omega \backslash \Sigma)$, we have

$$
\begin{aligned}
\int_{\Omega} u^{p} \eta \xi_{\varepsilon} d x & =-\int_{\Omega} u \Delta\left(\eta \xi_{\varepsilon}\right) d x \\
& =-\int_{\Omega} u\left(\eta \Delta \xi_{\varepsilon}+2 \nabla \eta \cdot \nabla \xi_{\varepsilon}+\xi_{\varepsilon} \Delta \eta\right) d x \\
& \leqq-\int_{\Omega} u\left(2 \nabla \eta \cdot \nabla \xi_{\varepsilon}+\xi_{\varepsilon} \Delta \eta\right) d x .
\end{aligned}
$$

Notice that both $\nabla \eta$ and $\Delta \eta$ vanish near $\Sigma$. Thus, by using (2.7) and Fatou's Lemma with (2.8), we get

$$
\int_{\Omega} u^{p} \eta d x \leqq-\int_{\Omega} u \Delta \eta d x<+\infty .
$$


Since $u \in C^{2}(\Omega \backslash \Sigma)$, this implies $u \in L_{\mathrm{loc}}^{p}(\Omega)$.

q.e.d.

Now we can get the lower bounds of $u$ satisfying (1.1) and (1.4).

LEMMA 2.2. Fix an arbitrary smooth domain $\Omega^{\prime}$ satisfying $\Sigma \Subset \Omega^{\prime} \Subset \Omega$. Then, for any $u$ satisfying (1.1) and (1.4),

$$
u(x) \geqq g(x)-C \quad x \in \bar{\Omega}^{\prime} \backslash \Sigma
$$

holds for a positive constant $C$.

Moreover $p<(n-m) /(n-m-2)$ holds if $(1.1)$ has a solution and if $m \leqq n-3$.

Proof. By (1.1), (1.4), (2.2), (2.3), (2.4), (2.5) and Lemma 2.1,

$$
0 \leqq-\Delta(u-g) \in L_{\mathrm{loc}}^{1}(\Omega), \quad u-g \in L_{\mathrm{loc}}^{1}(\Omega),
$$

and $u-g \in C^{2}(\Omega \backslash \Sigma)$. Thus we get (2.9) by the maximum principle.

Assume that $p \geqq(n-m) /(n-m-2)$ holds. Since $0 \leqq g(x) \leqq u(x)+C \in L_{\text {loc }}^{p}(\Omega)$, $g \in L_{\mathrm{loc}}^{q}(\Omega)$ holds with $q=(n-m) /(n-m-2)$. But this contradicts (2.4). Therefore $p<(n-m) /(n-m-2)$ holds if $m \leqq n-3$.

q.e.d.

Next we consider the upper bounds of $u$ satisfying (1.1) and (1.4). We recall (2.10). Therefore, by using $L^{1}$-elliptic regularity theory (see, for example, Benilan, Brezis and Crandall [2, Appendix, pp. 547-555]), we get the following.

LEMMA 2.3. For any $u$ satisfying (1.1) and (1.4), $u \in L_{\mathrm{loc}}^{q}(\Omega)$ holds for any $q \in[1, n /(n-2))(q \in[1, \infty)$ if $n=2)$.

LEMMA 2.4. Assume that $n=2$ and $m=1$. Then, for any $u$ satisfying (1.1) and (1.4), $u \in C^{0}(\Omega)$ holds.

Proof. We fix an arbitrary $q>p$. By (1.1), (1.4), (2.2) and Lemma 2.3, $-\Delta(u-g)=u^{p} \in L_{\mathrm{loc}}^{q / p}(\Omega)$. Thus, by using the Sobolev embedding, $u-g \in W_{\mathrm{loc}}^{2, q / p}(\Omega)$ $\subset C^{0}(\Omega)$. Since $g \in C^{0}(\bar{\Omega}), u \in C^{0}(\Omega)$ holds.

q.e.d.

We introduce the following function $h(x)$ for the case $n \geqq 3$.

$$
h(x)=\int_{\Sigma}|x-\sigma|^{-\tau} d \sigma \quad x \in \bar{\Omega} \backslash \Sigma \quad(n \geqq 3),
$$

where

$$
\tau= \begin{cases}m-2+(n-m-2) p & \text { (if } m \leqq n-3) \\ m-1 / 2 & \text { (if } m=n-2) \\ m-3 / 2 & \text { (if } m=n-1) .\end{cases}
$$

Then we have the following. 
LEMMA 2.5. Let $h(x)$ be as in (2.11). Assume that $n \geqq 3$ and that $p<(n-m)$ $/(n-m-2)$ if $m \leqq n-3$. Then

$$
\left\{\begin{array}{l}
\left.h(x) \in C^{0}(\bar{\Omega}) \quad \text { (if } m \geqq n-2 \text {, or if } m=n-3 \text { and } p \in(1,2)\right) \\
h(x) \leqq C|\log d(x)|+D \quad(\text { if } m=n-3 \text { and } p=2) \\
\left.h(x) \leqq C d(x)^{2-(n-m-2) p} \quad \text { (if } m \leqq n-4 \text {, or if } m=n-3 \text { and } p \in(2,3)\right),
\end{array}\right.
$$

$$
-\Delta h(x) \geqq \begin{cases}C d(x)^{-(n-m-2)} p & (\text { if } m \leqq n-3) \\ C d(x)^{-3 / 2} & \text { (if } m=n-2) \\ C d(x)^{-1 / 2} & \text { (if } m=n-1),\end{cases}
$$

and

$$
0 \leqq g(x)^{p} \leqq-C \Delta h(x)
$$

hold for $x \in \bar{\Omega} \backslash \Sigma$. Here $C$ and $D$ denote some positive constants.

Proof. We can immediately get (2.12) from (2.11) and Proposition A.2 in Appendix. Differentiating (2.11), we see

$$
-\Delta h(x)=\tau(n-2-\tau) \int_{\Sigma}|x-\sigma|^{-(\tau+2)} d \sigma \quad x \in \bar{\Omega} \backslash \Sigma,
$$

where

$$
\tau(n-2-\tau)=\left\{\begin{array}{l}
((n-m-2) p+m-2)(n-m-p(n-m-2)) \quad(\text { if } m \leqq n-3) \\
\frac{1}{2}\left(n-\frac{5}{2}\right) \quad(\text { if } m=n-2, \text { or if } m=n-1)
\end{array}\right.
$$

We recall that $m \geqq 1$ and $p>1$. Thus $\tau(n-2-\tau)>0$ holds. And we can easily get (2.13) from (2.15) and Proposition A.2. Furthermore (2.14) easily follows from (2.3) and (2.13).

q. e.d.

Now we have the following.

LEMma 2.6. Assume that $n \geqq 3$ and $p<n /(n-2)$. Fix an arbitrary smooth domain $\Omega^{\prime}$ satisfying $\Sigma \Subset \Omega^{\prime} \Subset \Omega$. Then, for any $u$ satisfying (1.1) and (1.4),

$$
u(x) \leqq g(x)+C h(x)+C \quad x \in \bar{\Omega}^{\prime} \backslash \Sigma
$$

holds for some positive constant $C$.

Proof. We fix an arbitrary $q \in(p, n /(n-2))$. Then, by Lemma 2.3, $u \in$ $L^{q}\left(\Omega^{\prime}\right)$. We put

$$
u=u_{0}+g \text {, }
$$

where $u_{0}$ satisfies 


$$
\left\{\begin{aligned}
-\Delta u_{0} & =u^{p} & & \text { in } \Omega^{\prime} \\
u_{0} & =u & & \text { on } \partial \Omega^{\prime} .
\end{aligned}\right.
$$

Since $0 \leqq u \in C^{2}\left(\bar{\Omega}^{\prime} \backslash \Sigma\right)$ and $u^{p} \in L^{q(0)}\left(\Omega^{\prime}\right)$ with $q(0)=q / p>1,(2.18)$ has a unique non-negative solution $u_{0} \in W^{2, q(0)}\left(\Omega^{\prime}\right)$. By the Sobolev embedding,

where

$$
u_{0} \in W^{2, q(0)}\left(\Omega^{\prime}\right) \subset L^{r(0)}\left(\Omega^{\prime}\right),
$$

$$
r(0)=n q(0) /(n-2 q(0))>n /(n-2) .
$$

Let $u_{1}$ be the solution of

$$
\left\{\begin{aligned}
-\Delta u_{1} & =\left(u_{0}\right)^{p} & & \text { in } \Omega^{\prime} \\
u_{1} & =u & & \text { on } \partial \Omega^{\prime} .
\end{aligned}\right.
$$

Since $u_{0} \in L^{r(0)}\left(\Omega^{\prime}\right)$ holds with $r(0)>n /(n-2)>p$, the same argument as above implies

where

$$
u_{1} \in W^{2, q(1)}\left(\Omega^{\prime}\right) \subset L^{r(1)}\left(\Omega^{\prime}\right)
$$

$$
\begin{aligned}
& q(1)=r(0) / p>1, \\
& r(1)=n q(1) /(n-2 q(1))>n /(n-2) .
\end{aligned}
$$

Furthermore, by (2.14), (2.17), (2.18) and (2.19),

and

$$
\begin{aligned}
-\Delta u_{0}=u^{p} & \leqq \\
& \left.\leqq u_{0}+g\right)^{p-1}\left(\left(u_{0}\right)^{p}+g^{p}\right) \leqq-C \Delta u_{1}-C \Delta h \quad \text { a.e. in } \Omega^{\prime}
\end{aligned}
$$

$$
u_{0}-C u_{1}-C h=-(C-1) u-C h \leqq 0 \quad \text { on } \partial \Omega^{\prime}
$$

hold, where $C>1$ denotes some positive constant. Thus, by the maximum principle,

$$
0 \leqq u_{0}(x) \leqq C_{1} u_{1}(x)+C_{1} h(x) \quad \text { a.e. in } \Omega^{\prime}
$$

hold for some positive constant $C_{1}>1$.

By (2.3) and (2.12), $h(x) \leqq$ const. $g(x)$ holds for $x \in \Omega^{\prime} \backslash \Sigma$. Observing this fact and (2.14), we have

$$
0 \leqq u_{0}(x) \leqq C_{j} u_{j}(x)+C_{j} h(x) \quad \text { a.e. in } \Omega^{\prime}
$$

for $j \geqq 1$, where $C$, denotes some positive constant and a sequence of functions $\left\{u_{j}\right\}_{j \geqq 1}$ is defined py letting $u_{j+1}$ be a unique solution of

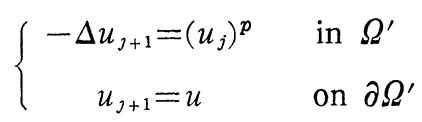


inductively for $j \geqq 0$. Furthermore,

$$
u_{j} \in W^{2, q(\jmath)}\left(\Omega^{\prime}\right) \subset L^{r(j)}\left(\Omega^{\prime}\right)
$$

hold for $j \geqq 0$, where $q(0)=q / p$ and

$$
q(j)=r(j-1) / p, \quad r(j)=n q(j) /(n-2 q(j))
$$

for $j \geqq 1$. Since $q>p$, we can easily see

$$
\begin{aligned}
1 / q(j)-2 / n & =2 / n(p-1)-(2 / n(p-1)-1 / q) p^{j} \\
& <2 / n(p-1)-(2 / n(p-1)-1 / p) p^{j} \\
& =\left(2-(n-(n-2) p) p^{\jmath-1}\right) / n(p-1)
\end{aligned}
$$

for $j \geqq 0$. We recall that $p<n /(n-2)$. Thus, $2 q(k)>n$ holds for some positive integer $k$. By the Sobolev embedding,

$$
u_{k} \in W^{2, q(k)}\left(\Omega^{\prime}\right) \subset C^{0}\left(\bar{\Omega}^{\prime}\right) .
$$

By (2.17), (2.20) and (2.21), we get (2.16).

q.e.d.

Now we are in a position to prove Theorem 2. From (2.3), Lemmas 2.2, $2.4,2.5$ and 2.6 . we can immediately get $(1.3)$ for the case $m \leqq n-2$ or the case $n=2$ and $m=1$. Therefore we only treat the case $m=n-1$ and $n \geqq 3$. We take an arbitrary $u$ satisfying (1.1) and (1.4). Then, by (2.2), Lemmas 2.5 and 2.6,

$$
-\Delta(u-g)=u^{p} \in L_{\mathrm{loc}}^{\infty}(\Omega), \quad u-g \in C^{2}(\Omega \backslash \Sigma) .
$$

Thus $u-g \in C^{0}(\Omega)$ holds. Since $g \in C^{0}(\bar{\Omega})$ by $(2.3), u \in C^{0}(\Omega)$. Now we get the desired Theorem 2.

\section{Existence of a solution}

Let $u$ be a solution of (1.1) satisfying (1.3). We take an arbitrary $\lambda>0$ and put $v=\lambda^{-1 /(p-1)} u, \beta=\lambda^{-p /(p-1)} \alpha$. Then $v$ satisfies

$$
\left\{\begin{array}{rlrl}
-\Delta v & =\lambda\left(v^{p}+\beta \delta_{\Sigma}\right) & & \text { in } \mathscr{D}^{\prime}(\Omega) \\
0 \leqq v \in C^{2}(\Omega \backslash \Sigma) & & (p>1, \lambda>0)
\end{array}\right.
$$

and the same bounds as in (1.3). Therefore we treat (3.1) hereafter. We put

$$
\bar{g}(x)=\int_{\Sigma} G(x, \sigma) \beta(\sigma) d \sigma .
$$

Then $0 \leqq \bar{g} \in C^{\infty}(\bar{\Omega} \backslash \Sigma)$ and $\bar{g}$ satisfies 


$$
\left\{\begin{array}{cc}
-\Delta \bar{g}=\beta \delta_{\Sigma} & \text { in } \mathscr{D}^{\prime}(\Omega) \\
\bar{g}=0 & \text { on } \partial \Omega
\end{array}\right.
$$

and the same properties as in (2.3), (2.4) and (2.5).

At first we construct a supersolution of (3.1).

LEMMA 3.1. Let $n \geqq 3$ and $h(x)$ be as in (2.11). Then there exist $\lambda>0$ and $\bar{v}$ satisfying

$$
\begin{gathered}
0 \leqq \bar{v}(x)=\lambda(\bar{g}(x)+h(x)) \in C^{\infty}(\bar{\Omega} \backslash \Sigma), \\
-\Delta \bar{v} \geqq \lambda\left(\bar{v}^{p}+\beta \delta_{\Sigma}\right) \quad \text { in } \mathscr{D}^{\prime}(\Omega) .
\end{gathered}
$$

Furthermore $\bar{v}$ satısfies the same bounds as in (1.3).

Proof. We only treat the case $m \leqq n-4$, since the other cases can be treated similarly. We put

$$
\bar{v}(x)=A(\bar{g}(x)+h(x)) \quad x \in \bar{\Omega} \backslash \Sigma,
$$

where $A>0$ is some constant which will be defined later. Then $\bar{v}$ satisfies (3.3) and

$$
-\Delta \bar{v}=A\left(\beta \delta_{\Sigma}-\Delta h\right) \quad \text { in } \mathscr{D}^{\prime}(\Omega) .
$$

By Lemma 2.2, we may assume that $p<(n-m) /(n-m-2)$ holds. Thus, by (2.3), (2.12), (2.13) and (3.5),

$$
\begin{aligned}
\bar{v}^{p}(x) & \leqq A^{p} 2^{p-1}\left(\bar{g}(x)^{p}+h(x)^{p}\right) \\
& \leqq A^{p} 2^{p-1}\left(C_{2} d(x)^{-(n-m-2) p}+C d(x)^{(2-(n-m-2) p) p}\right) \\
& =A^{p} 2^{p-1} d(x)^{-(n-m-2) p}\left(C_{2}+C d(x)^{(n-m-(n-m-2) p) p}\right) \\
& \leqq C_{3} A^{p} d(x)^{-(n-m-2) p} \leqq B A^{p}(-\Delta h(x))
\end{aligned}
$$

hold for $x \in \bar{\Omega} \backslash \Sigma$, where $C, C_{2}, C_{3}$ and $B$ denote some positive constant independent of $A$. By (3.6) and (3.7) we have

$$
-\Delta \bar{v} \geqq B A^{1-p} \bar{v}^{p}+A \beta \delta_{\Sigma} \quad \text { in } \mathscr{D}^{\prime}(\Omega) .
$$

Therefore we get (3.4) if we choose $\lambda=A=B^{1 / p}$.

q.e.d.

LemMA 3.2. Assume that $n=2$ and $m=1$. Then there exist $\lambda>0$ and $\bar{v}$ satisfying

$$
\left\{\begin{aligned}
-\Delta \bar{v} & \geqq \lambda\left(\bar{v}^{p}+\beta \delta_{\Sigma}\right) \quad \text { in } \mathscr{D}^{\prime}(\Omega) \\
0 & \leqq \bar{v} \in C^{0}(\bar{\Omega}) .
\end{aligned}\right.
$$

Proof. We put 


$$
w(x)=\int_{\Sigma}\left(\log \left(R|x-\sigma|^{-1}\right)\right)^{1 / 2} d \sigma \quad x \in \bar{\Omega} \backslash \Sigma .
$$

Here $R$ denotes the diameter of $\Omega$. By Proposition A.3 in Appendix, $0 \leqq w(x)$ $\in C^{0}(\bar{\Omega})$. Differentiating (3.9), we have

$$
-\Delta w(x)=4^{-1} \int_{\Sigma}|x-\sigma|^{-2}\left(\log \left(R|x-\sigma|^{-1}\right)\right)^{-3 / 2} d \sigma
$$

for $x \in \bar{\Omega} \backslash \Sigma$. Since $0<\log t \leqq 3 t^{1 / 3}$ hold for any $t>1$,

$$
0<\log \left(R|x-\sigma|^{-1}\right) \leqq 3 R^{1 / 3}|x-\sigma|^{-1 / 3}
$$

hold for any $x \in \bar{\Omega} \backslash \Sigma$ and any $\sigma \in \Sigma$. Thus, by Proposition A.2 in Appendix, we have

$$
-\Delta w(x) \geqq C_{1} \int_{\Sigma}|x-\sigma|^{-3 / 2} d \sigma \geqq C_{2} d(x)^{-1 / 2} \geqq C_{3}>0
$$

for $x \in \bar{\Omega} \backslash \Sigma$. Here $C_{1}, C_{2}$ and $C_{3}$ denote some positive constants.

We put

$$
\bar{v}(x)=A(\bar{g}(x)+w(x)) \quad x \in \bar{\Omega} \backslash \Sigma,
$$

where $A>0$ is some positive constant which will be defined later. Since $0 \leqq \bar{g}$ $\in C^{0}(\bar{\Omega}), 0 \leqq \bar{v} \in C^{0}(\bar{\Omega})$ and $\bar{v}(x)^{p} \leqq A^{p} C(x \in \bar{\Omega})$ hold for some positive constant $C$ independent of $A$. By (3.10) and (3.11), we have

$$
\begin{aligned}
-\Delta \bar{v} & =A\left(\beta \delta_{\Sigma}-\Delta w\right) \\
& \geqq A C_{3}+A \beta \delta_{\Sigma} \\
& \geqq A^{1-p} C_{3} C^{-1} \bar{v}^{p}+A \beta \delta_{\Sigma} \quad \text { in } \mathscr{D}^{\prime}(\Omega) .
\end{aligned}
$$

Therefore we get (3.8) if we choose $\lambda=A=\left(C_{3} / C\right)^{1 / p}$.

q.e.d.

Now we are in a position to prove Theorem 1. Let $\bar{v}$ be as in Lemma 3.1 (resp. Lemma 3.2) for the case $n \geqq 3$ (resp. $n=2$ and $m=1$ ). We define a sequence of functions $\left\{v_{j}\right\}_{\jmath \geqq 0}$ by $v_{0}=0$ and by letting $v_{\jmath+1}$ be a unique solution of

$$
\left\{\begin{aligned}
-\Delta v_{\jmath+1} & =\lambda\left(\left(v_{\jmath}\right)^{p}+\beta \delta_{\Sigma}\right) & & \text { in } \mathscr{D}^{\prime}(\Omega) \\
v_{\jmath+1} & =0 & & \text { on } \partial \Omega,
\end{aligned}\right.
$$

inductively. It is easy to see by induction that

$$
0 \leqq v_{j}(x) \leqq v_{j+1}(x) \leqq \bar{v}(x) \quad \text { a.e. in } \Omega
$$

for $j \geqq 0$. Thus, $v_{j}(x) \rightarrow v(x)(j \rightarrow \infty)$ a. e. in $\Omega$, which is a solution of (3.1) and satisfies

$$
\lambda \bar{g}(x)=v_{1}(x) \leqq v(x) \leqq \bar{v}(x) \quad \text { a. e. in } \Omega .
$$


From (2.3), (3.12), Lemmas 3.1 and 3.2 , we can easily get Theorem 1 .

\section{Appendix}

Let $\Omega, \Sigma$ be as in Introduction. At first we consider the following integral.

$$
I(x)=\int_{\Sigma}|x-\sigma|^{-s} d \sigma, \quad x \in \bar{\Omega} \backslash \Sigma \quad(s \in(0, \infty)) .
$$

Then we have the following.

Lemma A.1. We fix an arbitrary $a \in \Sigma$. Then there exists a small $\varepsilon>0$ such that

$$
\begin{aligned}
& C_{1} d(x)^{m-s} \leqq I(x) \leqq\left.C_{2} d(x)^{m-s}+D_{2} \quad \text { (if } s \neq m\right) \\
&-C_{1}(\log d(x))-D_{1} \leqq I(x) \\
&\left.\leqq-C_{2}(\log d(x))+D_{2} \quad \text { (if } s=m\right)
\end{aligned}
$$

hold for any $x \in B(\varepsilon ; a)$. Here $C_{1}, C_{2}, D_{1}$ and $D_{2}$ are some positive constants independent of $x, \varepsilon$ and $B(\varepsilon ; a)$ denotes the ball of radius $\varepsilon$ with the center $a$.

Proof. When $m=n-2$, the first inequality in (A.3) is proved in Vazquez and Veron [8, Lemma 2.3, pp. 129-130]. Therefore we use the same notations as in [8].

We fix $a \in \Sigma$ and set $B_{\eta}=B(\eta ; a), \Sigma_{\eta}=\Sigma \cap B_{\eta}$ for $\eta>0$. And we put

$$
I(x)=I_{1}(x)+I_{2}(x),
$$

where

$$
\begin{aligned}
& I_{1}(x)=\int_{\Sigma_{\eta}}|x-\sigma|^{-s} d \sigma \\
& I_{2}(x)=\int_{\Sigma_{\backslash \Sigma_{\eta}}}|x-\sigma|^{-s} d \sigma .
\end{aligned}
$$

There exists a local diffeomorphism from an open subset $G \subset \boldsymbol{R}^{n}$ onto $B_{\eta}$ such that $\Psi(0)=a$ and $\Psi(\omega)=\Sigma_{\eta}$ if $\omega=G \cap \boldsymbol{R}^{m}$. And the restriction $\bar{\Psi}$ of $\Psi$ to $\omega$ is a parametrization of $\Sigma_{\eta}$. If $y=(\bar{y}, \rho) \in \boldsymbol{R}^{m} \times \boldsymbol{R}^{n-m}, \bar{\Psi}(\bar{y})=\Psi(\bar{y}, 0)$. Thus we have

$$
I_{1}(x)=\int_{\omega} J(\bar{y})|x-\bar{\Psi}(\bar{y})|^{-s} d \bar{y},
$$

where

$$
J(\bar{y})=\left|\operatorname{det}\left\langle\frac{\partial \bar{\Psi}}{\partial \bar{y}_{i}}, \frac{\partial \bar{\Psi}}{\partial \bar{y}_{j}}\right\rangle\right|^{1 / 2} .
$$

As $\bar{\Psi}$ is a parametrization of $\Sigma_{\eta}$, we may assume that 


$$
\begin{gathered}
0<C_{1} \leqq J(\bar{y}) \leqq C_{2} \\
\left\{y \in \boldsymbol{R}^{n} ;|y| \leqq b\right\} \subset G \subset\left\{y \in \boldsymbol{R}^{n} ;|y| \leqq c\right\} .
\end{gathered}
$$

Here $C_{1}, C_{2}, b$ and $c$ denote some positive constants.

We take $\varepsilon>0$ such that $0<\varepsilon<\eta / 2$. And we take an arbitrary $x \in B_{\varepsilon}$ and write $x=\Psi(\bar{z}, \rho), \bar{z} \in \boldsymbol{R}^{m}, \rho \in \boldsymbol{R}^{n-m}$. If $\varepsilon$ is small enough,

$$
|\bar{z}|^{2}+|\rho|^{2}<(b / 2)^{2}
$$

holds. By (A.8) and (A.9), we have the following.

$$
\begin{aligned}
& \left\{\bar{y} \in \boldsymbol{R}^{m} ;|\bar{y}|<b / 2\right\} \subset\left\{\bar{y} \in \boldsymbol{R}^{m} ;|\bar{y}-\bar{z}|<b\right\} \\
& \left\{\bar{y} \in \boldsymbol{R}^{m} ;|\bar{y}-\bar{z}|<c\right\} \subset\left\{\bar{y} \in \boldsymbol{R}^{m} ;|\bar{y}|<2 c\right\}
\end{aligned}
$$

Summing up (A.5), (A.6), (A.7), (A.10) and (A.11),

$$
C_{3} I_{3}(x) \leqq I_{1}(x) \leqq C_{4} I_{4}(x)
$$

hold, where $C_{3}$ and $C_{4}$ are some positive constants and

$$
\begin{aligned}
& I_{3}(x)=\int_{0}^{b / 2} r^{m-1}\left(r^{2}+|\rho|^{2}\right)^{-s / 2} d r, \\
& I_{4}(x)=\int_{0}^{2 c} r^{m-1}\left(r^{2}+|\rho|^{2}\right)^{-s / 2} d r .
\end{aligned}
$$

Since $|\rho|<b / 2<2 c$ hold from (A.8) and (A.9),

$$
\begin{gathered}
I_{4}(x) \leqq \int_{0}^{|\rho|} r^{m-1}|\rho|^{-s} d r+\int_{|\rho|}^{2 c} r^{m-s-1} d r \\
\leqq \\
\quad \begin{array}{l}
\left.C|\rho|^{m-s}+D \quad \text { if } s \neq m\right) \\
C \log (2 c /|\rho|)+D \quad(\text { if } s=m),
\end{array} \\
\text { (A.14) } \quad I_{3}(x) \geqq \int_{0}^{|\rho|} r^{-s / 2}|\rho|^{-s} \int_{0}^{|\rho|} r^{m-1} d r=r^{-1} 2^{-s / 2}|\rho|^{m-s} \quad(s \in(0, \infty))
\end{gathered}
$$

and

$$
\begin{aligned}
I_{3}(x) & \geqq \int_{|\rho|}^{b / 2} r^{m-1}\left(r^{2}+|\rho|^{2}\right)^{-s / 2} d r \\
& \left.\geqq 2^{-s / 2} \int_{|\rho|}^{b / 2} r^{m-s-1} d r=2^{-m / 2} \log (b / 2|\rho|) \quad \text { (if } s=m\right)
\end{aligned}
$$

hold for some positive constants $C$ and $D$. By the way, from (A.6), 


$$
C_{5} d(x) \leqq|\rho| \leqq C_{6} d(x)
$$

hold for some positive constants $C_{5}$ and $C_{6}$.

By (A.12), (A.13), (A.14), (A.15) and (A.16), we can see that $I_{1}(x)$ satisfies the same bounds as in (A.2) and (A.3) for any $x \in B(\varepsilon ; a)$. On the other hand,

$$
0<I_{2}(x) \leqq(2 / \eta)^{s}|\Sigma|
$$

hold for any $x \in B(\varepsilon ; a)$, since $0<\varepsilon<\eta / 2$ and

$$
|x-\sigma| \geqq|\sigma-a|-|x-a| \geqq \eta-\varepsilon>\eta / 2
$$

hold for any $x \in B(\varepsilon ; a)$ and any $\sigma \in \Sigma \backslash \Sigma_{\eta}$. Therefore we get the desired results.

q.e.d.

Now we have the following.

Proposition A.2. Let $I(x)$ be as in (A.1).

i) If $s>m$,

$$
\begin{aligned}
& C_{1} d(x)^{m-s} \leqq I(x) \leqq C_{2} d(x)^{m-s} \quad x \in \bar{\Omega} \backslash \Sigma, \\
& I \in L^{q}(\Omega) \quad \text { for any } q \in(0,(n-m) /(s-m)), \\
& I \notin L^{q}(\Omega) \quad \text { for any } q \in[(n-m) /(s-m), \infty) .
\end{aligned}
$$

ii) If $s=m$,

$$
\begin{aligned}
& C_{1}|\log d(x)|-D_{1} \leqq I(x) \leqq C_{2}|\log d(x)|+D_{2} \quad x \in \bar{\Omega} \backslash \Sigma, \\
& I \in L^{q}(\Omega) \quad \text { for any } q \in(0, \infty) .
\end{aligned}
$$

iii) If $s<m, I \in C^{0}(\bar{\Omega})$.

Here $C_{1}, C_{2}, D_{1}$ and $D_{2}$ denote some positive constants.

Proof. At first we treat the case $s<m$. Fix an arbitrary $a \in \Sigma$. Then, by (A.2), $0<I(x) \leqq C$ holds for any $x \in B(\varepsilon ; a)$. Here $C$ is a positive constant independent of $x$ and $\varepsilon$. Thus, by using Fatou's Lemma, $I(x) \rightarrow I(a)$ as $x \rightarrow a$, Since $a \in \Sigma$ is arbitrary, we get iii).

Next we treat the case $s \geqq m$. Using the compactness of $\Sigma$, (A.2) and (A.3) remain valid in some neighbourhood of $\Sigma$. Since $I(x) \in C^{\infty}(\bar{\Omega} \backslash \Sigma)$, (A.2) and (A.3) remain valid in $\bar{\Omega}$.

Notice the following formula in Weyl [11].

$$
\int_{d(x)<\varepsilon} 1 d x=\left|B_{1}^{n-m}\right||\Sigma| \varepsilon^{n-m}+o\left(\varepsilon^{n-m+1}\right) \quad \text { as } \varepsilon \rightarrow 0 \text {. }
$$

Here $\left|B_{1}{ }^{n-m}\right|$ denotes the volume of the unit ball in $\boldsymbol{R}^{n-m}$.

From (A.2), (A.3) and (A.17), we can easily get i) and ii).

q. e. d.

Next we consider the following integral. 


$$
K(x)=\int_{\Sigma}\left(\log \left(R|x-\sigma|^{-1}\right)\right)^{s} d \sigma \quad x \in \bar{\Omega} \backslash \Sigma \quad(s \in(0, \infty)),
$$

where $R$ denotes the diameter of $\Omega$.

Since $|x-\sigma|<R$ for $\sigma \in \Sigma, x \in \bar{\Omega}$ and $0 \leqq \log t \leqq 2 s t^{1 /(2 s)}$ for $t \geqq 1$, we get

$$
0 \leqq K(x) \leqq(2 s)^{s} R^{1 / 2} \int_{\Sigma}|x-\sigma|^{-1 / 2} d \sigma .
$$

We fix an arbitrary $a \in \Sigma$. Then, by (A.1), (A.2) and (A.19),

$$
0 \leqq K(x) \leqq C_{2} d(x)^{m-1 / 2}+D_{2} \leqq C
$$

hold for any $x \in B(\varepsilon ; a)$. Here $C$ denotes some positive constant. Therefore the same argument as in the proof of Proposition A.2 yields the following.

Proposition A.3. Let $K(x)$ be as in (A.18). Then, $K(x) \in C^{0}(\bar{\Omega})$ holds for any $s \in(0, \infty)$.

\section{REFERENCES}

[1] P. Aviles, On isolated singularities in some nonlinear partial differential equations, Indiana Univ. Math. J., 32 (1983), 773-791.

[2] P. Benilan, H. Brezis and M.G. Crandall, A semilinear equation in $L^{1}\left(\boldsymbol{R}^{N}\right)$, Ann. Scuola Norm. Sup. Pisa, 2 (1975), 523-555.

[3] H. BREzis AND P.L. Lions, A note on isolated singularities for linear elliptic equations, Mathematical Analysis and Applications, Part A, Advances in Mathematics Supplementary Studies, 74, Academic Press, 1980, 263-266.

[4] H. BREZIS AND L. VERON, Removable singularities for some nonlinear elliptic equations, Arch. Ratiotal Mech. Anal., 75 (1980), 1-6.

[5] B. Gidas And J. Spruck, Global and local behaviour of positive solutions of nonlinear elliptic equations, Comm. Pure Appl. Math., 34 (1981), 525-598.

[6] P. L. Lions, Isolated singularities in semilinear problems, J. Differential Equations, 38 (1980), 441-450.

[7] J. SERrin, Isolated singularities of solutions of quasilinear equations, Acta Math., 113 (1965), 219-240.

[8] J. L. VAzQUez AND L. Veron, Singularities of elliptic equations with an exponential nonlinearity, Math. Ann., 296 (1984), 119-135.

[9] L. VERON, Singular solutions of some nonlinear elliptic equations, Nonlinear Anal., 5 (1981), 225-242.

[10] L. Veron, Singularités éliminable d'équatıons elliptics nonlinéaris, J. Differential Equations, 41 (1981), 87-95.

[11] H. WeYL, On the volume of tubes, Amer. J. Math., 61 (1939), 461-472.

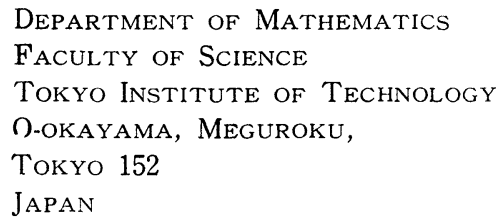

